\title{
A Low-Signal-to-Noise Ratio Estimation Algorithm for Multipath Channels
}

\author{
Baohai Yang ${ }^{1}$, Quanhui Ren ${ }^{2}$, Haisheng Li $^{1}$, Junkang Song ${ }^{1 *}$ \\ ${ }^{1}$ College of Physics and Electronic Engineering, Guangxi Normal University for Nationalities, Chongzuo 532200, China \\ ${ }^{2}$ Department of Electronic Engineering, Zhengzhou Railway Vocational and Technical College, Zhengzhou 451460, China
}

Corresponding Author Email: songjunkang@gxnun.edu.cn

https://doi.org/10.18280/isi.250213

Received: 17 November 2019

Accepted: 11 February 2020

\section{Keywords:}

multipath channel, periodic sequences, signal-to-noise ratio (SNR), low SNR estimation, white Gaussian noise (WGN)

\begin{abstract}
The traditional multipath channel algorithms cannot estimate low signal-to-noise ratio (SNR) in an accurate manner, which drags down the quality of the entire channel. To solve the problem, this paper puts forward a novel low SNR estimation algorithm for multipath channels. The core idea of the algorithm is as follows: the noise variance of received signals is estimated based on the good autocorrelation of periodical sequences; the signal amplitude is solved according to the statistical features of white Gaussian noise (WGN); finally, the SNR is estimated despite its low level. Matlab simulations show that our algorithm greatly outperformed the classic SNR estimation algorithms in accuracy, and its advantage increases with the decline in the SNR. The proposed algorithm has a great potential in the field of channel detection.
\end{abstract}

\section{INTRODUCTION}

Channel estimation aims to improve the accuracy of the received signals by estimating the state parameter and time/frequency domain response of the channel, and correcting and receiving the received data [1-3]. In practice, the quality of channel transmission is evaluated by the following parameters: signal-to-noise ratio (SNR), bit error rate (BER), frame error rate (FER), received signal strength (RSS), delay spread and Doppler frequency shift [4-5]. The BER and FER are the fundamental indices that directly reflect the transmission quality of a communication system. However, neither of them can track the channel state well, if the channel changes very quickly: the two indices require a massive amount of data to ensure their estimation accuracy, and consume a long time to output the estimates, exhibiting a poor real-time performance [6-8].

Among the channel quality metrics, the SNR has a great practical significance in measuring channel quality, thanks to its good real-time performance, and direct correlation with the BER and FER. Therefore, the SNR is an important parameter for channel estimation [9-10]. The SNR estimation is a key link in communication, because the SNR serves as the prior knowledge on many occasions, namely, channel equalization, power control, modulation identification, and iterative decoding of Turbo codes. The accuracy of the SNR estimation directly affects the performance of the communication system. If the SNR is not estimated accurately, the adaptive technologies in the system cannot operate normally in complex environments, such as coded modulation, multi-beam allocation, handover, and carrier recovery.

Many SNR estimation algorithms have been developed at home and abroad [11]. Some are data-aided (DA) and some are non-data-aided (NDA). The DA algorithms mainly include the maximum likelihood (ML) estimation and the least squares (LS) algorithm. The most representative DNA algorithms are frequency-domain estimation (FDE) and M2M4 estimation
[12-14]. Ishtiaq and Sheikh [15] predicted the SNR through ML estimation. Fu et al. [16] described the M2M4-based SNR estimation algorithm and derived its formulas. Through ML estimation, Ye et al. [17] forecasted the binary phase shift keying (BPSK) signal with white Gaussian noise (WGN). Du and $\mathrm{Xu}$ [18] derived the M2M4-based SNR estimation algorithm for real signal channel and complex signal channel with additive white Gaussian noise (AWGN); simulation results show that the algorithm estimates the SNR more accurately with the growing length of observed data, but performs not so well when the SNR is lower than $-5 \mathrm{~dB}$.

Based on M2M4 algorithm, Yang et al. [19] proposed a deep learning algorithm for SNR estimation, and verified the performance and robustness through experiments; the proposed algorithm is suitable for baseband signals and incoherent signals; compared with the M2M4, the proposed algorithm has a large application range of modulation method, and estimates the SNR in the middle layer in an accurate manner. Zhang et al. [20] introduced the derivation and details of the FDE: the noise energy is obtained through spectral segmentation, and used to calculate the SNR; the algorithm performs poorly if the SNR is very low. Han et al. [21] presents an SNR algorithm that estimates noise power based on the variance of the phase change in received signal, but the algorithm only applies to phase modulated signals. Targeting linearly modulated signals in the AWGN channel, Ali and Erçelebi [22] created an improved SNR estimation algorithm which does not need to estimate the total received signal power and enjoys a low complexity.

Ijaz et al. [23] put forward a novel NDA SNR estimation algorithm for BPSK and quadrature phase shift keying (QPSK) signals; the algorithm is less complex than the statistical moment-based method; the estimation performance is improved based on the constant amplitude of in-phase and quadrature components. Shbat and Tuzlukov [24] proposed a coding-aware joint estimation algorithm of carrier phase and SNR. Yong et al. [25] estimated the prior SNR based on 
modified sigmoid gain function, which overcomes the delay in decision-directed (DD) SNR estimation. For linear systems, Suliman et al. [26] developed a high-precision SNR estimation algorithm by producing received signals one after another. Queiroz et al. [27] probed into the moment method, a SNR estimation technique, in fading channel models. Wang et al. [28] proposed a priori SNR estimator based on harmonic regeneration, which effecitvely enhances the high-order harmonic components at a low SNR and promotes the performance of speech enhancement algoithtm. Ji et al. [29] improved the prior DD SNR estimation algorithm, and combined the improved algorithm with the noise estimation algorithm, which is based on the existence probability of speech; the combined method can estimate the power spectrum of noises well, and track rapidly changing noises in real time. Focusing on the data link communication of unmanned aerial vehicles (UAVs), Sun et al. [30] integrated convolutional neural network (CNN) and long short-term memory (LSTM) to estimate the SNR; as one of the earlies attempts to apply deep learning (DL) to SNR estimation, the CNN-LSTM algorithm has better accuracy than traditional SNR estimation algorithms. To predict the real-time SNR in the long run, Soleymani et al. [31] designed the adaptive longterm SNR estimation algorithm, whose SNR estimates only changes under non-transient variations in signals or noises; with low cost and fast update speed, the proposed algorithm is suitable for real-time speech processing.

This paper mainly explores the SNR estimation in multipath channels. Therefore, two classic SNR estimation algorithms, namely, M2M4 and FDE, were taken into account. On this basis, a low SNR estimation algorithm was developed for multipath channels. The proposed algorithm mainly estimates the low SNR, using the good autocorrelation of periodic sequences. Simulation results show that the proposed algorithm greatly outperformed M2M4 and FDE in the estimation accuracy of low SNR.

The remainder of this paper is organized as follows: Section 2 introduces the classic SNR estimation algorithm, and describes the principles of M2M4 and FDE; Section 3 details the principle, derivation and implementation of the proposed algorithm; Section 4 compares the performance of the proposed algorithm, M2M4 and FDE through Matlab simulations; Section 5 puts forward the conclusions.

\section{CLASSIC SNR ESTIMATION ALGORITHM}

The low pass of the received signals on multipath channel can be defined as:

$$
a(n)=\sum_{i} B_{i} e^{j \delta} y\left(n-\tau_{i}\right)+w(n)
$$

where, $y(n)$ is transmitted signals; $w(n)$ is the AWGN with a mean power of $\varphi^{2} ; \delta_{\mathrm{i}}, B_{\mathrm{i}}$ and $\tau_{\mathrm{i}}$ are the arrival phase, amplitude attenuation factor, and transmission delay of the i-th channel, respectively. The SNR of multipath channel can be expressed as:

$$
U_{S N}=\frac{P_{s}}{P_{w}}=\frac{\sum_{i} p_{s, i}}{\varphi^{2}}
$$

where, $p_{s}, p_{s, i}$ and $p_{w}$ are the total mean power, the mean power per channel and the mean power of noise, respectively. The short-term changes of $\delta_{i}, B_{i}$ and $\tau_{i}$ are negligible, due to the slow fading of the channel [32].

\subsection{Principle of M2M4}

In the WGN, the second-order statistic is the only nonzero element, i.e. the only noisy element. Thus, the SNR of the MPSK signals in a WGN-containing channel can be expressed as:

$$
\hat{U}_{S N}=\frac{\overline{2 m_{2}^{2}-m_{4}}}{m_{2}-\overline{2 m_{2}^{2}-m_{4}}}
$$

where, $m_{2}$ and $m_{4}$ are second-order moment and fourth-order moment, respectively:

$$
m_{2}=\frac{1}{N} \sum_{n=0}^{N-1}|a(n)|^{2}, m_{4}=\frac{1}{N} \sum_{n=0}^{N-1}|a(n)|^{4}
$$

where, $a(n)$ is a sequence of received signals $(n=0,1, \ldots, N-1)$.

On the upside, the M2M4 is a simple algorithm. On the downside, the algorithm cannot estimate low SNR accurately, under the effect of noises [33].

\subsection{Principle of FDE}

The FDE estimates the SNR based on the noise energy, which is obtained by spectral segmentation. Let $A(n)$ be the Npoint discrete Fourier transform of $a(n)$, and $Q=N / Z$ be the number of frequency points in each of the $Z$ equal-width segment of channel bandwidth. Then, the mean noise power and the SNR can be respectively expressed as:

$$
\begin{aligned}
\hat{p}_{w} & =\frac{1}{Q} \min \left(\sum_{k=i Q}^{(i+1) Q-1}|A(k)|^{2}\right), i=0,1 \cdots, Z-1 \\
& \hat{U}_{S N} \\
= & \frac{\hat{p}_{S}}{\hat{p}_{W}}=\frac{\hat{p}_{a}-\hat{p}_{W}}{\hat{p}_{W}} \\
= & \frac{\frac{1}{N} \sum_{k=0}^{N-1}|A(k)|^{2}-\frac{1}{Q} \min \left(\sum_{k=i Q}^{(i+1) Q-1}|A(k)|^{2}\right)}{\frac{1}{Q} \min \left(\sum_{k=i Q}^{(i+1) Q-1}|A(k)|^{2}\right)}
\end{aligned}
$$

It can be seen that the FDE is a simple way to accurately estimate the SNR of narrow-band signals in a channel with AWGN. If the SNR is low, however, this algorithm will have difficulty in SNR estimation, for the signal spectrum is affected by the noise spectrum [34].

\section{LOW SNR ESTIMATION ALGORITHM FOR MULTIPATH CHANNEL}

\subsection{Basic principle}

The proposed algorithm mainly estimates the SNR based on 
the periodic sequences that carry no data [35]. The periodic sequence $x(n)$ with $L$ and $N$ periods can be expressed as:

$$
x(n)=\sum_{k=0}^{N-1} s(n-k L)
$$

where, $s(n)$ is the sequence of intervals for principal values $(n=0,1, \ldots, L-1)$. The autocorrelation function of $x(n)$ can be expressed as:

$$
U_{\tau}=\sum_{n=0}^{L-1} s(n) s(n+\tau)
$$

Since $x(n)$ and $U_{\tau}$ are both periodic and have the same period, we have:

$$
U(-\tau)=U(K L-\tau), K \text { is an integer }
$$

The accuracy of SNR estimation increases with the autocorrelation of $x(n)$. As mentioned before, the short-term changes of $\delta_{i}, B_{i}$ and $\tau_{i}$ are negligible, due to the slow fading of the channel. Here, the short-wave multipath slow-fading channel is observed for $T \leq 50 \mathrm{~ms}$. Let $V$ be the velocity of $x(n)$. Then, $N=T V / L$ within $T$.

\subsection{Algorithm derivation}

The widely used two-path channel (short-wave multipath slow fading channel) was adopted to derive the formulas of the proposed algorithm. Suppose that the signals on the two path are synchronized, and the transmission delay $\tau$ is known (path 2 signals are delayed by $\tau$ code elements compared to path 1 signals).

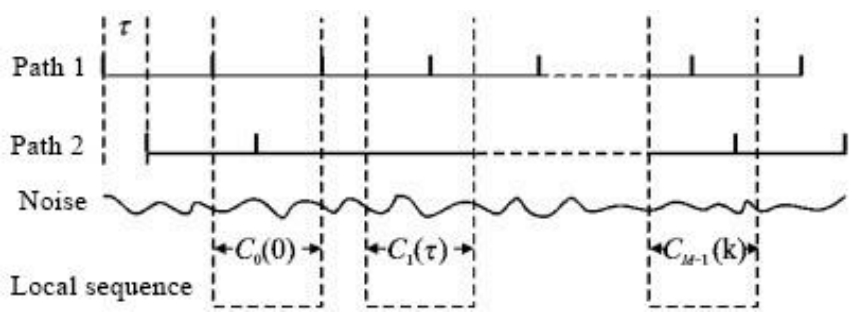

Figure 1. Local sequence and received signals

As shown in Figure 1, the signal amplitudes of the two paths are denoted as $A_{1}$ and $A_{2}$, respectively. The local sequence (the sequence of principal value intervals) was used to perform a sliding operation on the received signals. The sliding operation lasts $M$ periods, sliding over 1 code element per period. Then, the the k-th correlation value in the $\mathrm{m}$-th period can be expressed as:

$$
C_{m}(k)=A_{1} U(k)+A_{2} U(k-\tau)+E_{m}(k)
$$

where, $C_{m}(0)$ and $C_{m}(\tau)$ are the correlation values synchronized with path 1 and path 2 in the m-th period, respectively; $m=0,1, \ldots, M-1 ; k=0,1, \ldots, L-1$. Let $E_{m}(k)$ be a Gaussian random variable with the mean of zero and variance of $\varphi^{2}$. Then, the mean, variance and second-order moment of the variable can be respectively described as:

$$
\left\{\begin{array}{l}
W\left[E_{m}(k)\right]=0 \\
D\left[E_{m}(k)\right]=\sum_{n=0}^{L-1} s^{2}(n) D\left(w_{m l+k+n}\right)=L \varphi^{2} \\
W\left[E_{m}(k)^{2}\right]=D\left[E_{m}(k)\right]+W\left[E_{m}(k)\right]=L \varphi^{2}
\end{array}\right.
$$

The mean signal power and the noise variance must be obtained to estimate the SNR. Since the signal amplitudes of the two paths are denoted as $A_{1}$ and $A_{2}$, respectively, the mean of $C_{m}(0)$ and $C_{m}(\tau)$ can be derived from the statistical features of the WGN:

$$
\left\{\begin{array}{l}
W\left[C_{m}(0)\right] \\
=W\left[A_{1} U(0)+A_{2} U(-\tau)+E_{m}(0)\right] \\
=A_{1} U(0)+A_{2} U(-\tau) \\
W\left[C_{m}(\tau)\right] \\
=W\left[A_{1} U(\tau)+A_{2} U(0)+E_{m}(\tau)\right] \\
=A_{1} U(\tau)+A_{2} U(0)
\end{array}\right.
$$

Suppose $\overline{C(0)}=W\left[C_{m}(0)\right]$ and $\overline{C(\tau)}=W\left[C_{m}(\tau)\right]$. The following can be derived from formula (11):

$$
\begin{aligned}
& A_{1}=\frac{\overline{C(0)} U(0)-\overline{C(\tau)} U(-\tau)}{U(0)^{2}-U(\tau) U(-\tau)} \\
& A_{2}=\frac{\overline{C(\tau)} U(0)-\overline{C(0)} U(-\tau)}{U(0)^{2}-U(\tau) U(-\tau)}
\end{aligned}
$$

Thus, the mean signal power can be solved as:

$$
p_{x}=p_{x, 1}+p_{x, 2}=A_{1}^{2}+A_{2}^{2}
$$

If the mean of $C_{m}{ }^{2}(k)$ is known, the noise variance $\varphi^{2}$ can be obtained. The mean $C_{m}{ }^{2}(k)$ can be obtained by formula (11):

$$
\begin{aligned}
& W\left[C_{m}^{2}(k)\right] \\
& =W\left\{\left[A_{1} U(k)+A_{2} U(k-\tau)+E_{m}(k)\right]^{2}\right\} \\
& =W\left\{\left[A_{1} U(k)+A_{2} U(k-\tau)^{2}\right\}\right. \\
& +2\left[A_{1} U(k)+A_{2} U(k-\tau)\right] W\left[E_{m}(k)\right]+W\left[E_{m}^{2}(k)\right] \\
& =\left[A_{1} U(k)+A_{2} U(k-\tau)\right]^{2}+L \phi^{2}
\end{aligned}
$$
Let

The correlation values can improve the estimation accuracy.

$$
\overline{C^{2}}=\frac{1}{L} \sum_{k=0}^{L-1} W\left[C_{m}^{2}(k)\right]
$$

Thus

$$
\overline{C^{2}}=\frac{1}{L} \sum_{k=0}^{L-1}\left[A_{1} U(k)+A_{2} U(k-\tau)^{2}\right]+L \varphi^{2}
$$

The mean noise power can be obtained as:

$$
\varphi^{2}=\frac{\overline{C^{2}}-\frac{1}{L} \sum_{k=0}^{L-1}\left[A_{1} U(k)+A_{2} U(k-\tau)\right]^{2}}{L}
$$


The SNR at the receiving end can be described as:

$$
U_{\mathrm{SN}}=\frac{A_{1}^{2}+A_{2}^{2}}{\varphi^{2}} \frac{\left.L\{\overline{C(0)} U(0)-\overline{C(\tau)} U(-\tau)]^{2}+[\overline{C(\tau)} U(0)-\overline{C(0)} U(\tau)]^{2}\right\}}{\left[U(0)^{2}-U(\tau) U(-\tau)\right]^{2}\left\{\overline{C^{2}}-\frac{1}{L} \sum_{k=0}^{L-1}\left[A_{1} U(k)+A_{2} U(k-\tau)\right]^{2}\right\}}
$$

\subsection{Algorithm implementation}

The SNR estimation requires the length of the periodic sequence to be sufficiently long. This requirement is difficult to satisfy in actual applications. The arithmetic means of $C_{m}(0)$, $C_{m}(\tau)$ and $C_{m}{ }^{2}(k)$ can be approximately as $\overline{C(0)}, \overline{C(\tau)}$ and $\overline{C^{2}}$, respectively:

$$
\left\{\begin{array}{l}
\overline{C(0)}=\frac{1}{M} \sum_{m=0}^{M-1} C_{m}(0) \\
\overline{C(\tau)}=\frac{1}{M} \sum_{m=0}^{M-1} C_{m}(\tau) \\
\overline{C^{2}}=\frac{1}{L} \sum_{k=0}^{L-1}\left[\frac{1}{M} \sum_{m=0}^{M-1} C_{m}^{2}(k)\right]
\end{array}\right.
$$

The SNR can be estimated by substituting formula (20) into formula (19). The derivation and implementation demonstrate that the proposed algorithm applies to SNR estimation for channels with two or more paths.

\section{SIMULATION VERIFICATION}

To verify its correctness and superiority, the proposed algorithm was subjected to Matlab simulations, in comparison with M2M4 and FDE. The performance of each algorithm was measured by the mean SNR estimate, normalized mean square error (NMSE), and the bias probability. The closer the mean SNR estimate is to the actual SNR, the better the estimation performance. The NMSE reflects the deviation of the estimate from the actual value; the smaller the NMSE, the better the estimation accuracy. The bias probability refers to the probability that the difference between SNR estimate and actual SNR falls in the bias $b$; this metric reflects the estimation stability in a long-term statistical sense; here the bias $\mathrm{b}$ was set to $1 \mathrm{~dB}$ and $2 \mathrm{~dB}$; the greater the bias probability, the more stable the estimation.

The simulation parameters were configured as: the number of code elements, 496; $L=31$ and $M=16$. Figures 2, 3, and 4 compare the mean SNR estimates, the NMSEs, and the bias probabilities of the three algorithms, respectively.

As shown in Figure 2, the mean SNR estimates of all three algorithms were close to the actual value, when the SNR was greater than $5 \mathrm{~dB}$, indicating that the algorithms have similar performance in high SNR estimation. When the SNR was below $5 \mathrm{~dB}$, the proposed algorithm achieved the best performance, as its mean SNR estimate was basically the same as the actual value, while the mean SNR estimates of FDE and M2M4 deviated greatly from the actual value. Overall, our algorithm is much superior to the FDE and M2M4 in low SNR estimation.

As shown in Figure 3, when the SNR was lower than $1 \mathrm{~dB}$, our algorithm achieved the minimum NMSE; the advantage of our algorithm over M2M4 and FDE in NMSE increased with the decline of the SNR. This means our algorithm has the best estimation accuracy of low SNR.

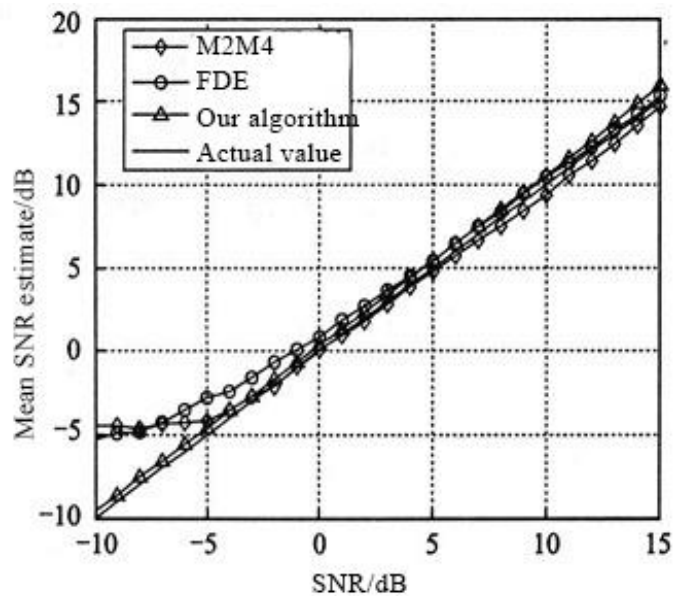

Figure 2. Comparison of mean SNR estimates

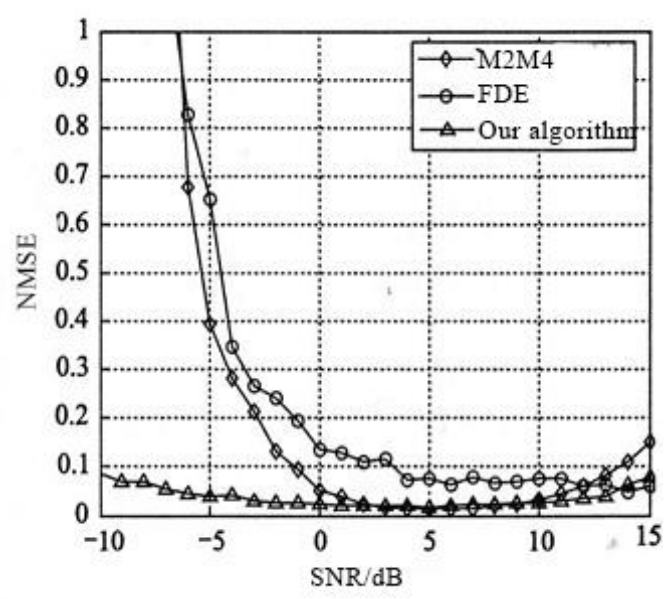

Figure 3. Comparison of NMSEs

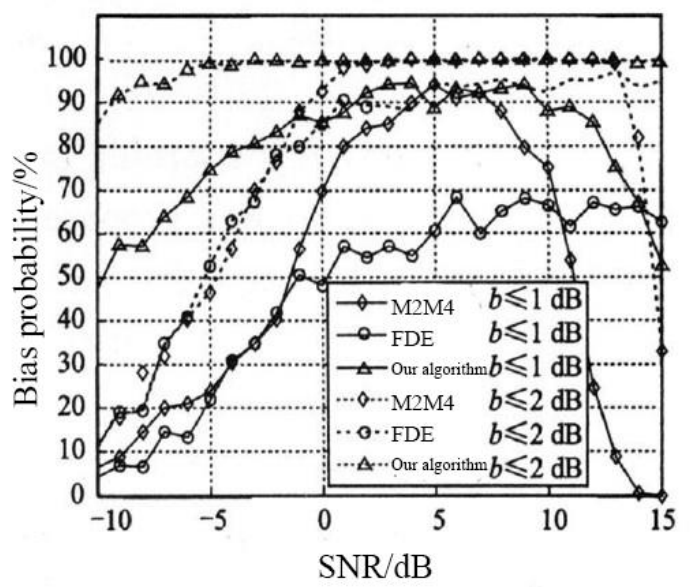

Figure 4. Comparison of bias probabilities

As shown in Figure 4, under the bias of $2 \mathrm{~dB}$, the bias probability of our algorithm was above $90 \%$ in the whole range of SNRs, while the bias probabilities of the two contrastive algorithms were far smaller, when the SNR was below $0 \mathrm{~dB}$. Under the bias of $1 \mathrm{~dB}$, our algorithm had a larger bias probability than the other two algorithms. When the SNR fell between -10 and $-5 \mathrm{~dB}$, our algorithm had a higher bias probability under the bias of $1 \mathrm{~dB}$ than the M2M4 and the FDE. The above results indicate that our algorithm has better stability than the two contrastive algorithms. 


\section{CONCLUSIONS}

This paper proposes a low SNR estimation algorithm for multipath channels. Based on the good autocorrelation of periodic sequences, the proposed algorithm fully utilizes the statistical features of the WGN to compute the signal amplitudes of two paths. On this basis, the mean noise power and SNR were estimated. Matlab simulations show that our algorithm outperformed two classic SNR estimation algorithms, namely, M2M4 and FDE, in the estimation accuracy of low SNR; the advantage of our algorithm over M2M4 and FDE in NMSE increased with the decline of the SNR. Therefore, our algorithm can be widely applied in the field of channel detection.

\section{ACKNOWLEDGMENT}

This work was supported in part by the Research Foundation of health department of Jiangxi Province under Grant 20183016, in part by the Science Foundation of Jiangxi Provincial Department of Education under Grant GJJ170954, in part by the Science Foundation of Guangxi Normal University for Nationalities under Grant 2019FG008.

\section{REFERENCES}

[1] Akbarpour-Kasgari, A., Ardebilipour, M. (2018). Probability-based pilot allocation for MIMO relay distributed compressed sensing channel estimation. EURASIP Journal on Advances in Signal Processing, 18. https://doi.org/10.1186/s13634-018-0539-7

[2] Abdou, A., Abdo, A., Jamoos, A. (2019). Overlay cognitive radio based on OFDM with channel estimation issues. Wireless Personal Communications, 108(2): 1079-1096. https://doi.org/10.1007/s11277-019-06455-2

[3] Ren, G., Chang, Y., Zhang, H. (2008). SNR estimation algorithm based on the preamble for wireless OFDM systems. Science in China Series F: Information Sciences, 51(7): 965-974. https://doi.org/10.1007/s11432-0080063-8

[4] Baghaki, A., Champagne, B. (2018). Joint frequency offset, time offset, and channel estimation for OFDM/OQAM systems. EURASIP Journal on Advances in Signal Processing, 4. https://doi.org/10.1186/s13634-017-0526-4

[5] Sahoo, H.K., Mohanty, B., Patnaik, B. (2018). Block and fast block sparse adaptive filtering for outdoor wireless channel estimation and equalization. Wireless Personal Communications, $\quad$ 98(3): 3003-3019. https://doi.org/10.1007/s11277-017-5013-6

[6] Chiluveru, S.R., Tripathy, M. (2019). Low SNR speech enhancement with DNN based phase estimation. International Journal of Speech Technology, 22(1): 283292. https://doi.org/10.1007/s10772-019-09603-y

[7] Yao, R., Zeng, Z., Zhu, P. (2016). A priori SNR estimation and noise estimation for speech enhancement. EURASIP Journal on Advances in Signal Processing, 101. https://doi.org/10.1186/s13634-016-0398-z

[8] Lerga, J., Sucic, V., Boashash, B. (2011). An efficient algorithm for instantaneous frequency estimation of nonstationary multicomponent signals in low SNR. EURASIP Journal on Advances in Signal Processing,
725189. https://doi.org/10.1155/2011/725189

[9] Uwaechia, A.N., Mahyuddin, N.M., Ain, M.F., Latiff, N.M.A., Za'bah, N.F. (2019). Compressed channel estimation for massive MIMO-OFDM systems over doubly selective channels. Physical Communication, 36: 100771. https://doi.org/10.1016/j.phycom.2019.100771

[10] Hua, J., Meng, L., Xu, Z., Wang, D. (2007). A new method for SNR and Doppler shift estimation in wireless propagations. Journal of Electromagnetic Waves and Applications, 21(15): 2431-2441. https://doi.org/10.1163/156939307783134317

[11] Jeya, R., Amutha, B. (2019). Optimized semiblind sparse channel estimation algorithm for MU-MIMO OFDM system. Computer Communications, 146: 103-109. https://doi.org/10.1016/j.comcom.2019.07.018

[12] Mecklenbräuker, C.F., Gerstoft, P., Zöchmann, E., Groll, H. (2020). Robust estimation of DOA from array data at low SNR. Signal Processing, 166, 107262. https://doi.org/10.1016/j.sigpro.2019.107262

[13] Li, Y., Song, T., Gurusamy, M., Yu, C., Kam, P.Y. (2018). Enhanced adaptive DA-ML carrier phase estimator and its application to accurate laser linewidth and SNR estimation. Optics Express, 26(12): 1481714831. https://doi.org/10.1364/OE.26.014817

[14] Chen, F., Kang, Y., Yu, H., Ji, F. (2014). Non-data-aided ML SNR estimation for AWGN channels with deterministic interference. EURASIP Journal on Wireless Communications and Networking, 45. https://doi.org/10.1186/1687-1499-2014-45

[15] Ishtiaq, N., Sheikh, S.A. (2016). Maximum likelihood SNR estimation for QAM signals over slow flat fading Rayleigh channel. TIIS, 10(11): 5365-5380. https://doi.org/10.3837/tiis.2016.11.009

[16] Fu, Y., Zhu, J., Wang, S., Zhai, H. (2014). Robust nondata-aided SNR estimation for multilevel constellations via Kolmogorov-Smirnov test. IEEE Communications Letters, $18(10)$ : https://doi.org/10.1109/LCOMM.2014.2356473

[17] Ye, X., Zhang, A., Zheng, G., You, L., Gao, X. (2018). Multicell multiuser massive MIMO channel estimation and MPSK signal block detection applying twodimensional compressed sensing. EURASIP Journal on Wireless Communications and Networking, 238: 1-10. https://doi.org/10.1186/s13638-018-1252-9

[18] Du, L.J., Xu, J. (2005). The research of the SNR estimation the research of the SNR estimation algorithm using second-order and four-order moments. Journal of Shandong University of Technology (Science and Technology), 19(6): https://doi.org/10.13367/j.cnki.sdgc.2005.06.013

[19] Yang, K., Huang, Z., Wang, X., Wang, F. (2019). An SNR estimation technique based on deep learning. Electronics, $\quad 8(10)$ : https://doi.org/10.3390/electronics8101139

[20] Zhang, K.F., Guo, Y., Qi, Z.S. (2017). A parameter estimation algorithm for multiple frequency-hopping signals based on sparse Bayesian method. Mathematical Problems in Engineering, 6129120. https://doi.org/10.1155/2017/6129120

[21] Han, S., Zhang, P., Shi, F., Wang, F.Y. (2017). An SNRadaptive relaying algorithm for multi-antenna cooperative networks. China Communications, 14(10): 228-236. https://doi.org/10.1109/CC.2017.8107646

[22] Ali, A.K., Erçelebi, E. (2019). An M-QAM signal 
modulation recognition algorithm in AWGN channel. Scientific Programming, 6752694. https://doi.org/10.1155/2019/6752694

[23] Ijaz, A., Awoseyila, A.B., Evans, B.G. (2009). Improved SNR estimation for BPSK and QPSK signals. Electronics letters, $\quad 45(16)$ : 858-859. https://doi.org/10.1049/el.2009.1759

[24] Shbat, M., Tuzlukov, V. (2019). Primary signal detection algorithms for spectrum sensing at low SNR over fading channels in cognitive radio. Digital Signal Processing, 93: 187-207. https://doi.org/10.1016/j.dsp.2019.07.016

[25] Yong, P.C., Nordholm, S., Dam, H.H. (2013). Optimization and evaluation of sigmoid function with a priori SNR estimate for real-time speech enhancement. Speech Communication, 55(2): 358-376. https://doi.org/10.1016/j.specom.2012.09.004

[26] Suliman, M.A., Alrashdi, A.M., Ballal, T., Al-Naffouri, T.Y. (2017). Snr estimation in linear systems with gaussian matrices. IEEE Signal Processing Letters, 24(12): $1867-1871$ https://doi.org/10.1109/LSP.2017.2757398

[27] Queiroz, W.J., Almeida, D.B., Madeiro, F., Cardoso, J.V.D.M., Pereira, D.F., Alencar, M.S. (2018). New closed-form expressions for SNR estimates of Nakagami fading channels by the method of moments. Telecommunication Systems, 69(3): 321-333. https://doi.org/10.1007/s11235-018-0440-6

[28] Wang, J., Yang, C.C., Mo, J.Y., Wang, D.Z., Wang, X.X. (2018). A priori SNR estimator based on harmonic regeneration. Computer Engineering and Applications, 54(7): 44-48. https://doi.org/10.3778/j.issn.10028331.1611-0366

[29] Ji, H.F., Jia, H.R., Wang, Y. (2019). Speech enhancement method for improving phase spectrum compensation. Computer Engineering and Applications, 55(8): 48-52. https://doi.org/10.3778/j.issn.10028331.1805-0454

[30] Sun, Y.H., Zeng, G.Q., Liu, C.H., Zhang, D.N. (2019). SNR estimation algorithm for UAV data link based on deep learning. Journal of Beijing University of Aeronautics and Astronautics, 45(9): 1855-1863. https://doi.org/10.13700/j.bh.1001-5965.2018.0724

[31] Soleymani, R., Selesnick, I.W., Landsberger, D.M. (2019). ALTIS: A new algorithm for adaptive long-term SNR estimation in multi-talker babble. Computer Speech \& Language, 58: 231-246. https://doi.org/10.1016/j.csl.2019.05.001

[32] Sastry, K.S., Babu, M.P. (2013). Non data aided SNR estimation for OFDM signals in frequency selective fading channels. Wireless personal communications, 70(1): 165-175. https://doi.org/10.1007/s11277-0120686-3

[33] Gao, M.Y., Gu, J., Chai, H. (2018). Research into the application of SNR estimation algorithms under out-field condition. Shipboard Electronic Countermeasure, 41(2): 80-84. https://doi.org/10.16426/j.cnki.jcdzdk.2018.02.018

[34] Moazzeni, T., Jiang, Y., Chen, T.D. (2016). Dataclassification-based SNR estimation for linearly modulated signals. Computers \& Electrical Engineering, 56: $85-95$. https://doi.org/10.1016/j.compeleceng.2016.09.01

[35] Krishnamurthy, S.D., Sabat, S.L. (2016). Blind SNR estimation for M-ARY Frequency Shift Keying signal using covariance technique. AEU-International Journal of Electronics and Communications, 70(10): 1388-1394. https://doi.org/10.1016/j.aeue.2016.07.012 